\title{
Las publicaciones periódicas de bibliotecología en los países en desarrollo
}

\author{
Adolfo Rodríguez Gallardo \\ Director General de Bibliotecas /UNAM \\ Investigador del CUIB
}

\section{INTRODUCCIÓN}

En 1981, durante la reunión de la IFLA EN Lipzig, tuve la oportunidad de asistir a una sesión de la Mesa Redonda sobre publicaciones periódicas en bibliotecología. En ella Richard M. Dougherty leyó un documento en el que proponía emprender un programa de capacitación para los responsables de este tipo de publicaciones en contacto y colaboración con las Asociaciones de Bibliotecarios. Al finalizar la reunión, comente el trabajo de Dougherty, le sugerí que quizás obtendría mejor resultado si se capacitaba a los editores que trabajan en las escuelas de bibliotecarios, en mi opinión en esas instituciones se produce la mayor cantidad de títulos. Reconozco que esta hipótesis no estaba basada en ninguna información sólida, era el producto de la experiencia mexicana que es la más cercana y la que mejor conozco. Prometí enviar a Dougherty un listado de publicaciones que probaran mi hipótesis. A continuación describo la metodología seguida para analizar la información disponible, así como los resultados obtenidos.

\section{METODOLOGÍA}

Con el fin de probar la hipótesis se decidió utilizar la publicación de la FID número 532, en la que se incluyen 950 títulos producidos en 79 países en desa- 
rrollo'. Las publicaciones fueron agrupadas en ocho categorías por tipo de editor:

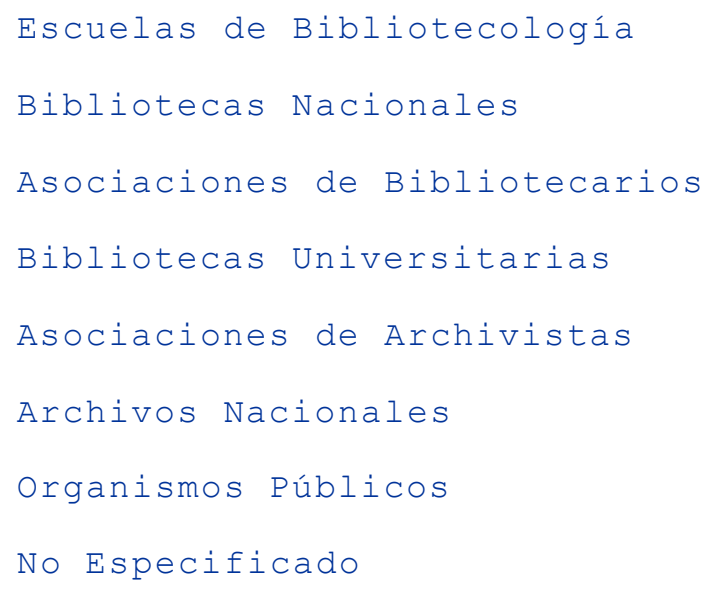

Es conveniente aclarar que por organismos públicos comprendimos a oficinas gubernamentales como, ministerios, secretarías, consejos e institutos que formen parte del gobierno, excluyendo a universidades, archivos nacionales, escuelas de bibliotecología y bibliotecas. Dentro de la categoría de los no especificados se incluyeron títulos cuya información disponible no era suficiente para identificar al organismo que los editó.

Se hizo una división por regiones para poder analizar con mayor profundidad la procedencia de las publicaciones periódicas, tanto por su origen geográfico como por el tipo de editores. El criteriogeográfico fue muy amplio y se decidió usar como marco para la organización de la información los continentes. Esto nos llevó a contar con cinco divisiones:

\author{
América Latina \\ Caribe inglés y holandés \\ Africa \\ Asia \\ Oceanía
}

En el caso de los países de América, éstos se dividieron entre aquellos países propiamente latinoamericanos, es decir, los que fueron colonias de España, Portugal o Francia, y los que fueron colonias británicas u holandesas que se 
agruparon en el apartado destinado al Caribe. Posteriormente se unió la información pues no se obtuvieron datos que justificaran su separación. La causa inicial de la división había sido, buscar si existía alguna diferencia sustantiva que permitiera distinguir una herencia bibliotecaria transmitida de las metrópolis a las colonias.

En el caso de Oceanía, se agrupó este continente con Asia aunque de antemano se reconoce que algunos países de la primera zona difícilmente pueden ser considerados asiáticos. Es necesario explicar que esos países son en su mayoría desarrollados y al incluir algún dato como el de Fiji, el resto de la información no se altera.

Resultó muy difícil separar a los países árabes pues no era deseable una división sobre el Oriente medio debido a la dificultad en determinar sus fronteras. Por Africa se entiende todos los países que están en ese continente, separados por el canal de Suez y el Mar Rojo de otros países del oriente medio que fueron incorporado a Asia.

En este análisis se excluyeron todas las publicaciones de las distintas regiones, independientemente de si están publicadas por algún organismo o agencia internacional aunque se presentan por separado en el cuadro respectivo. El criterio usado para seleccionar esos títulos es el lugar de publicación y no su contenido o la lengua en que fueron publicadas.

Para determinar los países que debían ser considerados naciones en desarrollo se utilizó la Guía del Tercer Mundo $1979^{2}$ y se aplicaron los criterios seguidos por esa publicación.

\section{RESULTADOS}

Las dos grandes áreas productoras de publicaciones periódicas en bibliotecología son Asia y América Latina con un número relativamente equivalente, pues Asia producía 59 títulos en tanto que Latinoamérica publicaba 61. En las tres áreas son las asociaciones de bibliotecarios las que publican mayor número de títulos con una producción del 50\% del total de publicaciones en todas las regiones. Los datos parciales por región indican que, mientras que en América Latina las asociaciones producen ligeramente más del $40 \%$ de los títulos de la zona, en Asia prácticamente es el 50\%. En Africa la relación es diferente ya que el 90\% de las publicaciones son producidas por asociaciones de bibliote- 
carios. La hipótesis de Dougherty es correcta y lo más lógico es aceptar que cualquier acción que se emprenda en este sentido sea en colaboración con las asociaciones. Es necesario dar, no obstante, un paso importante hacia un análisis cualitativo de los materiales ya que algunas de esas publicaciones son noticieros y hojas informativas de carácter muy local y que carecen de aportaciones sistemáticas y metodológicas a la bibliotecología.

En la comparación de datos se percibe diferencias y similitudes interesantes. Por ejemplo, el número de títulos producidos por las bibliotecas universitarias de Asia y de América Latina es muy parecido, correspondiendo cinco a la primera región y cuatro a la segunda. Las escuelas de bibliotecología de Asia y Latinoamérica producen algunos materiales que representan el 5\% entre las dos zonas, pero América Latina produce el doble con cuatro títulos, dos de ellos mexicanos, lo cual ayuda a entender el supuesto de que las escuelas de bibliotecología eran mayores productoras. Asia produce dos y Africa ninguno.

Las bibliotecas nacionales latinoamericanas son más prolíficas, producen seis títulos, mientras que las de Africa sólo producen uno y las de Asia tres. Los organismos públicos latinoamericanos son los editores del $20 \%$ de los títulos de la región y del $10 \%$ del total de las publicaciones, mientras que en Africa no publican ningún título y sólo cinco en Asia.

En América Latina los archivistas producen casi el 15\% de las publicaciones de la región; tres títulos son editados por las asociaciones de profesionales y cinco por los archivos nacionales. Las asociaciones de archivistas de Africa y Asia, cuando las hay, no producen ningún título, así como tampoco los archivos nacionales de Africa, solamente un archivo nacional de un país asiático edita una publicación en este campo. Posiblemente por desconocimiento de mi parte, al no poder identificar a los editores, encontré que en Asia y especialmente en la india hay un número importante de publicaciones periódicas editadas por organismos no especificados.

Los dos grandes productores de publicaciones periódicas son Brasil en América Latina y la India en Asia. En los dos casos las asociaciones de bibliotecarios son las principales productoras de materiales. 
Los organismos internacionales con sede en las tres regiones estudiadas producen en total 18 títulos; como puede verse en el cuadro 2, se sigue la misma pauta que se mostró en la concentración general de datos que se encuentran en el cuadro 1.

En total, los organismos internacionales publican 18 títulos en las tres regiones en que hemos dividido nuestro estudio. Las proporciones son muy parecidas a las correspondientes a los títulos incluidos en el cuadro 1, resultado de los esfuerzos propios de cada país. Lo que si es muy importante destacar es que ninguna de las publicaciones periódicas editadas por los organismos internacionales estaba incluida en los servicios de índice y resúmenes. Podría deducirse que lo que producen no es relevante a nivel internacional, o que hay una preferencia por los trabajos con cobertura nacional sobre los que intentan estudiar una región.

Una de las posibilidades de analizar la importancia de los contenidos de las publicaciones periódicas, es estudiarlos mediante el análisis de los diversos títulos en las publicaciones secundarias especializadas en bibliotecología. Antes de continuar con este trabajo hay que señalar dos aspectos: que la producción del Tercer Mundo no llega a la publicaciones secundarias por falta de una adecuada promoción y difusión; y que cuandollagan los índices internacionales no los tienen en cuenta por desconocer las lenguas en que se publican - considerar que el contenido de las publicaciones del Tercer Mundo no pueden contribuir de modo importante al desarrollo de nuestra especialidad.

Las publicaciones periódicas incluidas en obras secundarias están consignadas en la publicación 532 de la FID; se utilizaron las abreviaturas del cuadro de publicaciones periódicas indizadas; países que las publicaron y tipos de editor diferente. Las abreviaturas para identificar a los servicios de índice y resúmenes son las siguientes:

CALL Current Awarness - Library Literature 


\author{
IB Informationsdienst Bibliothekswesen \\ ISA Information Science Abstracts \\ LL Library Literature \\ LISA Library and Information Science Abstracts \\ RzI Referativnyi Zhurnal 59, Informatika
}

\title{
INDICES, LISTA DE PUBLICACIONES SECUNDARIAS
}

El cuadro sobre publicaciones incluidas en índices internacionales por zonas geográficas confirma la hipótesis general de que partimos. Si bien es cierto que la principal fuente de producción siguen siendo las asociaciones de bibliotecarios, también lo es que, la mitad de la producción proviene de otras fuentes, lo mismo que en el cuadro 1 sobre tipo de publicaciones por regiones. Lo importante es destacar que la producción entre lo publicado y lo indizado es mayor en las revistas no patrocinadas por las Asociaciones de Bibliotecarios. Las escuelas de bibliotecología producen en total seis publicaciones, tres de ellas indizadas, lo cual significa un 50\%, en tanto que las asociaciones publicaron 69 títulos y solamente se indizan 16, es decir, 25\% de lo publicado. Una posible explicación sería la falta de calidad del material incluido en tales publicaciones, la inadecuada distribución de las publicaciones, y el hecho de que se trate de noticieros y publicaciones que no contienen estudios.

Cabe destacar que tanto las publicaciones de las asociaciones de bibliotecarios de Africa como las de las bibliotecas universitarias de Asia están indizadas en la misma proporción. Las asociaciones africanas producen 13 títulos y hay indizadas cinco, lo cual representa un 45\% de la producción; en el caso de las bibliotecas universitarias asiáticas se producen cinco títulos y se indizan tres, cifra que representa más del $50 \%$.

Es interesante destacar que de los materiales producidos por las asociaciones de archivistas y por los archivos nacionales, esto es, tres títulos de las primeras y seis de los segundos ninguno está indizado en los servicios secundarios que utilizamos en este análisis. 
Por las observaciones anteriores, así como por el contenido de los diversos cuadros estadísticos, podemos deducir que las publicaciones periódicas que se editan en los países del Tercer Mundo son pocas y no tienen grandes posibilidades de ser incluidas en las publicaciones secundarias ya que de 135 títulos producidos solamente 27 son analizados por los servicios de índices y resúmenes, o sea que solamente se analiza el 20\% de las revistas.

Las publicaciones secundarias incluyen las revistas del siguiente modo:

LISA Incluye 23 de esas publicaciones, es decir, un 85\% del total, con lo cual logra ser la publicación secundaria de mayor cobertura.

ISA Y RzI cubren cuatro títulos cada una, que sólo representa respectivamente un $15 \%$ de la cobertura.

Ll cubre 15 títulos, casi el 55\% del total de títulos incluidos en publicaciones secundarias.

CALl analiza 13 títulos que representan casi el 50\% de las publicaciones del Tercer Mundo cubiertas por servicios de índice y resúmenes.

IB Analiza dos títulos que significan un modesto 8\% del total.

Como podemos observar por lo anterior, el índice de mayor cobertura es LISA, seguido de LL y posteriormente de CALL. Los otros servicios incluyen sólo marginalmente las publicaciones del Tercer Mundo.

La explicación de por qué LISA tiene una cobertura tan alta nos la proporciona Bárbara J. Buckley cuando dice que existe un acuerdo entre LISA y la British Library para usar sus colecciones ${ }^{3}$, lo cual explica que LISA esté en posibilidad de cubrir todas estas publicaciones ya que no representa ningún gasto para ésta. 
NOTAS

1.Barbara J. Buckley, "The coverage of Library: information science periodical from developing countries by the major abstracting and indexing services". En : IFLA General Conference. Round Table of Editors of Library Journals (48 th : 1982 : Montreal). Montreal: s.n., 1982.p 1.

2. Guía del tercer mundo. 1979. México : Periodistas del Tercer Mundo, 1979.

3. Grazyna Janzing. Library, documentation and archive, serials. Compiled by Grazyna Janzing: edited by K. R. Brow. $4^{\text {th }}$ ed. La Haya : FID, 1975. V, 203p. ( FID publication: 532)

CUADROS

$C \cup A D R O 1$

PUBLICACIONES POR TIPO OE EDITOR Y REGION

L. D I I O R

Escuelas de Bibliotecologia

Bibliotecas Nacionales

Asociación de Bibliotecas

Bibliotecas Universitarias

Asaciaciones de Archivistas

Archivos Nacionales

Drganismos Públiços

No Especificados

T I I \& L E S
ANERICA LAIIMA AFRICA ASIA TOTAL

$\begin{array}{rrrr}4 & 2 & 6 \\ 6 & 1 & 3 & 10 \\ 25 & 13 & 30 & 68 \\ 4 & 1 & 5 & 10 \\ 3 & & & 3 \\ 5 & & 1 & 6 \\ 12 & & 5 & 17 \\ 2 & & 13 & 15 \\ 51 & 15 & 59 & 135\end{array}$




\section{CUA}

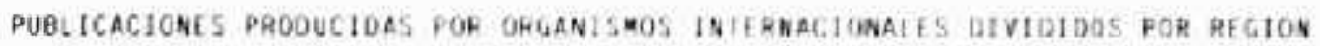

$$
\text { ANERTELTINA AHEILA ASIA TOTAL }
$$

$y$

C. If $A$ \& $B$ B $A$ I

PUBLICACIONES PLH]OOICAS INCLUHOAS IN INOJCES INIERNACIONALES POA AEGION

$$
\text { IDI OR AMIRLLLITINA AFRLCA ASIA TOLAL. }
$$

Escuelas de Bibliotecologia $\quad$ ? $\quad 1 \quad 3$

Bibliotecas Nacionales 1

Asociaciones de Bibliotection 79 to

Bibliotecas liniveritarias. 14

Asociaciones de Archivistas

Archivos Nacionales

Organismos Püblicos $\quad 1 \quad 1 \quad 2$

No Espesificos 1

$\begin{array}{lllll}10 \mathrm{AL} & 7 & 15 & 27\end{array}$

$[U \wedge \square R O$,

PUALICALIDNES PERIOOICAS INDIZADAS

PAISES QUE LAS PUALICAN Y TIDO DE IDTIOAES

Nomare de IA puBticarion IHDS. MDA PAIS EOITOR

Docusentación Bibliotucelfigica 1.55 Argentina Bu

Eastern Librarian

IISA Gangladesh AO

Ciencia da informacad

$\operatorname{LISA}$

G7. Erasil OP

Revista da fscald te Bibliote cononia da UFMG

ISA Brasil E8

Revista de Bibllatecanasia de

Brasil (RBB)

isA

11

ISA Brasil AB

Ghana Library Jeurna?

IIs

it. Gnana

A $B$ 


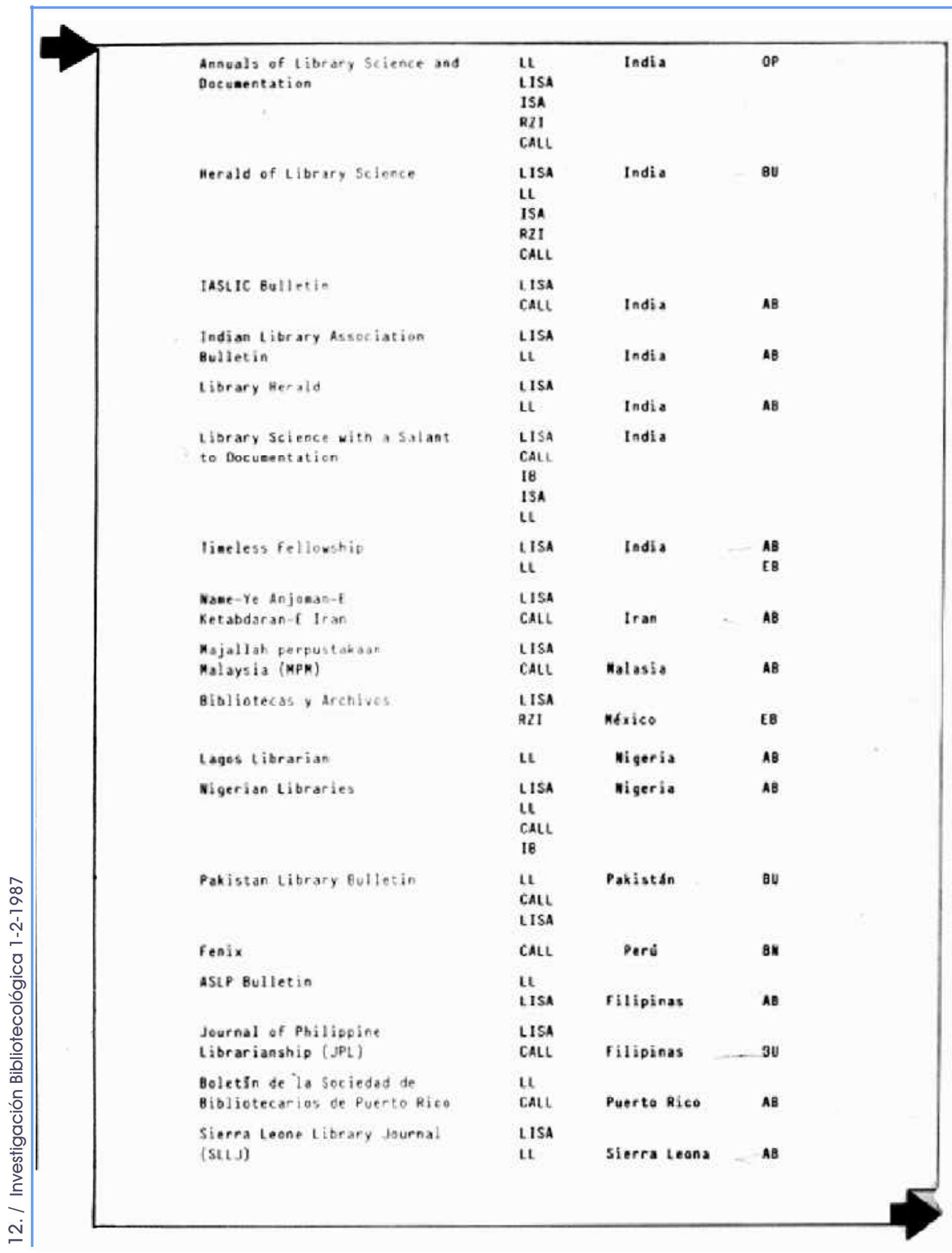




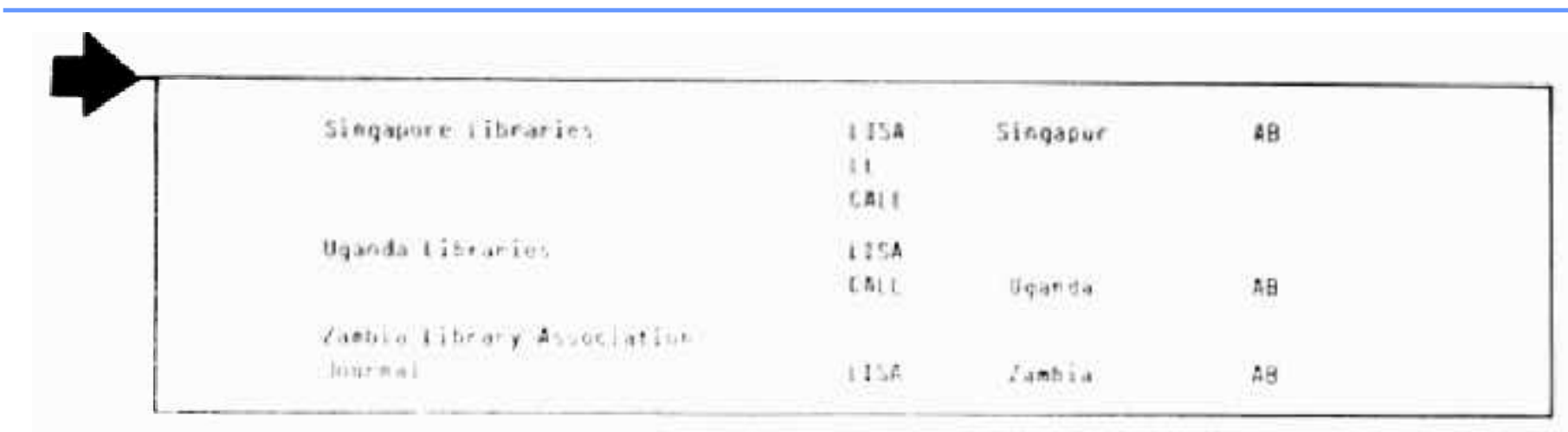

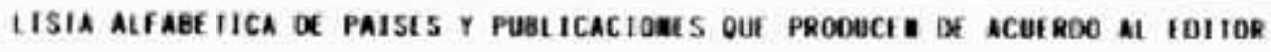

\begin{tabular}{|c|c|c|c|c|c|c|c|c|c|}
\hline Moubre del paits & E $\theta$ & 8 & $A B$ & B u & 11 & A : & DP & $?$ & Iatal \\
\hline ARGE NIINA & 1 & & 3 & 2 & 1 & 1 & & & 8 \\
\hline Bt $:$ VGI ADESH & & & 1 & & & & & & 1 \\
\hline BAREADOS & & & 1 & & & & & & 1 \\
\hline BRASII & 1 & & 11 & & & 1 & 7 & 1 & 21 \\
\hline CHILt & & & & & & & 1 & & 1 \\
\hline COLOMEIA & & & $?$ & & t & & I & 1 & 5 \\
\hline CDREA & & 1 & 3 & & & & & & 4 \\
\hline COSIA RICA & & & 3 & & & 2 & & & 2 \\
\hline CUBA & & 1 & & & & & 3 & & 4 \\
\hline EGLPTO & & & $t$ & & & & & & 1 \\
\hline EL SAL VADUR & & 1 & 1 & & 1 & 1 & & & 4 \\
\hline ETIOPIA & & & 1 & & & & & & 1 \\
\hline FILIPINAS & 1 & 1 & 3 & 1 & & & & & b \\
\hline$f 1 u$ & & & 1 & & & & & & 1 \\
\hline GHANA & & & 1 & & & & & & 1 \\
\hline GUAIEMALA & & 1 & & & & & & & 1 \\
\hline GUYANA & & & $t$ & & & & & & 1 \\
\hline INOIA & 1 & & 8 & 2 & & 1 & & 1 & 26 \\
\hline INDONESIA & & & 1 & & & & & & 2 \\
\hline IRAN & & & 2 & 1 & & & & & 3 \\
\hline JOROAN IA & & & $!$ & & & & & & 1 \\
\hline KENIA & & & 1 & & & & & & 1 \\
\hline
\end{tabular}




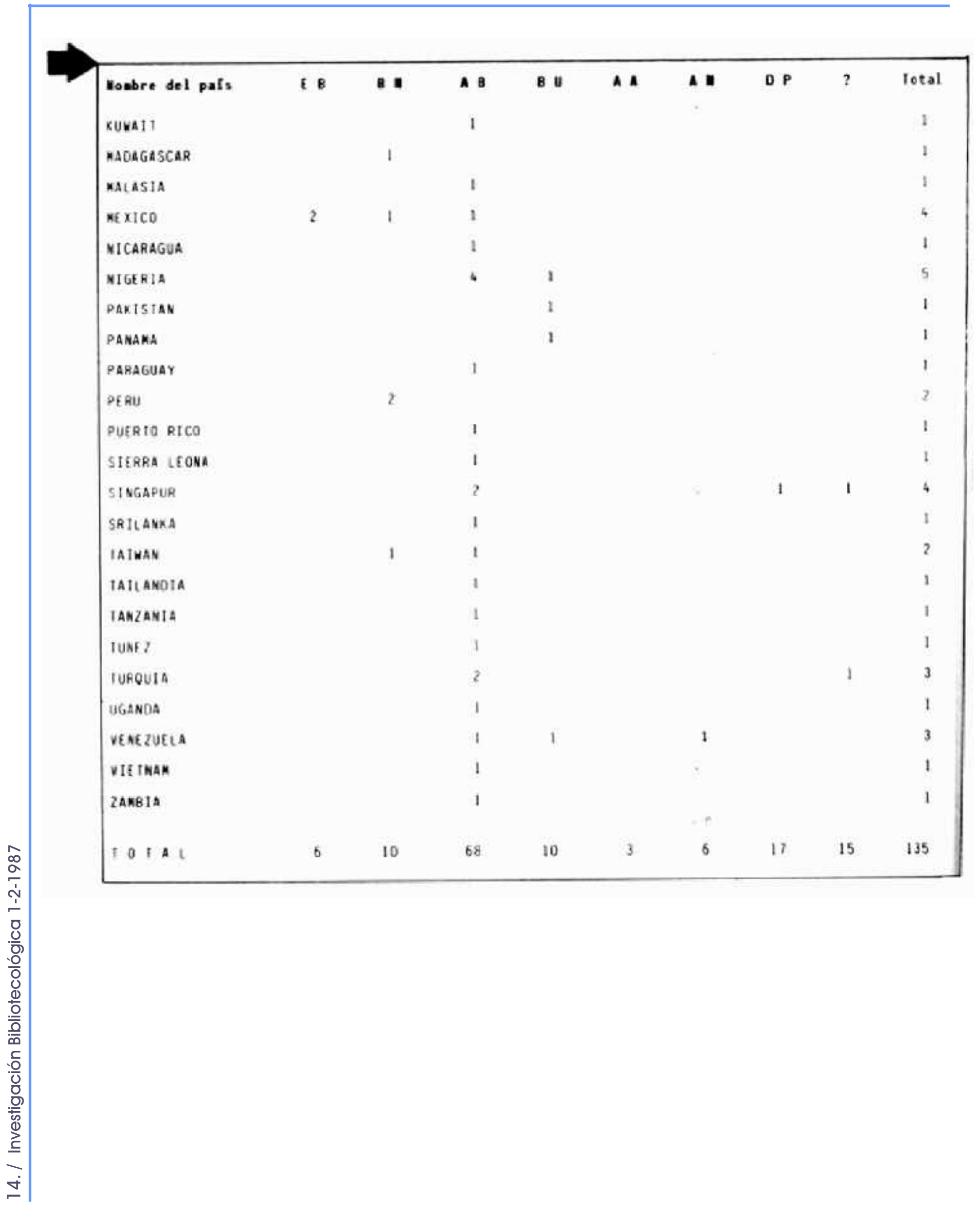

